\title{
Expansion and evaporation of hot nuclei: Comparison between semi-classical and quantal mean-field approaches
}

\author{
D. Lacroix ${ }^{1}$, Ph. Chomaz ${ }^{1}$ \\ (1)G.A.N.I.L., B.P. 5027, F-14076 Caen Cedex 5, France.
}

September 4, 2018

\begin{abstract}
We present a general discussion of the mean field dynamics of finite nuclei prepared under extreme conditions of temperature and pressure We compare the prediction of semi-classical approximation with complete quantum simulations. Many features of the dynamics are carefully studied such as the collective expansion, the evaporation process, the different time-scale... This study points out many quantitative differences between quantum and semi-classical approaches. Part of the differences are related to numerical features inherent in semi-classical simulations but most of them are a direct consequence of the non treatment of nuclei as quantal objects. In particular, we show that because of a too strong damping in semi-classical approaches the expansion of hot nuclei is quenched and the speed of the collective motion reduced.
\end{abstract}

PACS: 24.10.Cn, 05.60+w, 03.65.Sq, 25.70.Gh

Keywords: Mean-field, transport theory, TDHF, Vlasov, Hot nuclei. 


\section{Introduction}

Semi-classical methods are widely used to describe the nuclear dynamics. In particular, considerable progresses have been made in semi-classical transport theory of Heavy Ion Collisions with the simulation of Boltzmann type, in which an averaged Pauli-blocked collision term is added on top of a meanfield evolution [1, 2, 3]. More recently, following the Boltzmann-Langevin (BL) theory, a stochastic term has been also introduced to take into account the strong fluctuations of observables [9]. These approaches (BUU and BL) are of great interest not only because they are numerically tractable but also because they seem to be globally in good agreement with experimental data.

As far as quantum features are concerned, semi-classical approaches essentially take into account the exclusion principle through the Pauli blocking in nucleon-nucleon collisions. Thus, many properties of the nucleus which are due to the quantum nature of the nuclear systems 10, might not be correctly described in semi-classical approximations. For example, delocalization of wave-packets, wave dynamics, barrier transmission... are absent from semi-classical approaches, it is thus of great interest to check if the loss of these quantal features of nucleons does not change the evolution of a highly excited nucleus. The validity of semi-classical theories was already discussed several times and some qualitative differences have already been pointedout 11. However, we want to present a systematic quantitative comparison for the desexcitation of nuclei under extreme conditions.

In this paper, we compare the evolution of a hot and compressed (or diluted) nucleus in quantal calculation with the results of a semi-classical simulation. Our aim is to bring quantitative information about the possible role of the quantal nature of the nucleons and about the accuracy of semi-classical approaches. Since, most of the discussed phenomena are already present at the mean-field level, we will restrict ourselves to a comparison between time dependent Hartree-Fock (TDHF) methods and its semi-classical counterpart: the Vlasov dynamics. Moreover, we will consider a simple scenario of the evolution of a hot spherical compound nucleus and we will analyze evaporation of particles, dynamics of collective motion, time-scales...

In section 2, we present the considered scenario together with the various

\footnotetext{
${ }^{1}$ These approaches are often referred under different acronyms BUU, VUU, BNV 四, 司, 6. 6... In this article we will use BUU (for Boltzmann-Ueling-Ulenbeck) which is the one initially proposed by Bertsch et al [1, 2, 3]
} 
ingredients entering in the mean-field approximation. We will see that some of the differences between quantum and semi-classical simulations could be related to the finite sampling of the phase-space density, necessary in semiclassical methods. This numerical sampling of the phase-space generates a spurious finite range term in the semi-classical mean-field which was not in the original quantal description. This term modifies the properties of a finite piece of nuclear matter. When the quantum potential has a finite range component one may try to reduce the spurious effects by artificially reducing the range of the interaction used in the semi-classical simulations. We will show that this method is hardly a good approximation especially due to the density dependence of the various term in the interaction. In order to disentangle the role of this numerical artifact from a real difference between a quantal and a semi-classical treatment, a third type of simulation will be displayed: we will consider a TDHF simulation where a surface term has been added to the interaction in order to mimic the semi-classical numerical range.

In section 3, we present results concerning the expansion dynamics of initial excited nuclei with different compression or dilatation.

As expected, the semi-classical simulation seems to be a better approximation of the TDHF simulation where the surface term has been introduced. Nevertheless, many differences remain, showing limits of semi-classical calculations. For instance, evaporation rate, dynamics of the collective motion and associated time-scales are different. The condition under which a quantum system will expand toward low density regions will be finally discussed.

\section{A scenario to compare mean-field approaches.}

A good benchmark for a comparison between semi-classical and quantum dynamics is provided by the model case introduced by D. Vautherin et al [13]. Indeed, they consider an excited spherical nucleus at various initial temperature and with various density profiles. Then, they follow the evolution in the TDHF framework. The argument in favor of the use of a mean-field evolution is that the nucleon-nucleon collisions are not important for the dynamics of such thermalized system. Indeed, in the case of an equilibrated expanding source the gain and the loss terms in the collision part of the BUU approach almost cancel. 


\subsection{The interaction}

We will study the self-consistent mean field evolution of a spherical nucleus under extreme conditions. In the first part of this article we shall consider a density dependent effective two-body interaction:

$$
\hat{V}(1,2)=t_{0} \delta\left(\hat{\overrightarrow{r_{1}}}-\hat{\overrightarrow{r_{2}}}\right)+t_{3} \rho\left(\frac{\hat{\overrightarrow{r_{1}}}+\hat{\overrightarrow{r_{2}}}}{2}\right)^{\sigma} \delta\left(\hat{\overrightarrow{r_{1}}}-\hat{\overrightarrow{r_{2}}}\right),
$$

which is a simplified version of the more general Skyrme force, but has been shown to be sufficient for the study of the expansion dynamics 13. In the mean-field approach, for spin-isospin saturated systems, this interaction corresponds to a local potential energy

$$
E[\hat{\rho}](r, t)=\frac{3}{8} t_{0} \rho^{2}(r, t)+\frac{1}{16} \rho^{2+\sigma}(r, t),
$$

Note that, in the second part of this article, we shall add more terms to this interaction in order to take into account the finite range of the nuclear forces. Many of the useful results and derivations considering mean-field for a Skyrme force are derived in ref. 114.

For our numerical applications, we have taken $t_{0}=-1000 \mathrm{MeV} \mathrm{fm}^{3}$, $t_{3}=15000 \mathrm{MeV} \mathrm{fm}^{6}$ and $\sigma=1$ (see ref. [13]). In the infinite nuclear matter, this potential is globally in agreement with some more complicated parametrization 15]: this particular force yields to the following saturation point properties: $E / A=-14 \mathrm{MeV}$, a saturation density $\rho_{0}=0.15 \mathrm{fm}^{-3}$ and a compression modulus $K_{\infty}=336.2 \mathrm{MeV}$. This value corresponds to a rather stiff equation of state. Another interesting quantity is the "zero temperature spinodal density" , this is the density where the compressibility drops to zero at zero temperature. For the considered force, this density is equal to $0.1 \mathrm{fm}^{-3}$.

\subsection{Constrained mean-field initialization}

In simulation of mean field type, the usual way for preparing systems at different temperatures and with different shapes $\prec \hat{Q} \succ$, where $\hat{Q}$ is the observable measuring the deformation, is to perform a constrained meanfield calculation with a constraining field $\lambda \hat{Q}$. For instance, $\hat{Q}$ can be the square radius operator and $\lambda$ is computed in order to obtain the desired 
square radius $\prec \hat{r}^{2} \succ$ or equivalently the desired compression factor $\eta=$ $\left(\prec \hat{r}^{2} \succ_{0} / \prec \hat{r}^{2} \succ\right)^{3 / 2}$ (where $\prec \hat{r}^{2} \succ_{0}$ is the RMS of the ground state at zero temperature). We will also define the compression using the average density $(\rho)$ in a sphere of radius $2 \mathrm{fm}$ around the center of the nucleus. The value of $2 \mathrm{fm}$ is small enough compared to the RMS to avoid the bias of the surface and big enough to take into account not only the central s-orbitals but also others in order to focus on collective effects.

The temperature can be either fixed arbitrary to study isothermal initial conditions or can be defined in order to get a given energy $\prec \hat{H} \succ$. This method is well adapted to generate isothermal initial conditions however when the nucleus is strongly compressed, the self-consistency of the meanfield may lead to some shape modification such as the apparition of hollow structures. Moreover, it appears rather difficult to generate diluted nuclei since negative $\lambda$ values leads to unbound systems.

\subsection{Isoentropic scaling initialization}

In order to overcome the limitations of the constrained mean-field approach and to be able to reach both high or low densities we have considered an alternative procedure based on the scaling of the nucleus. Since the scaling preserves the entropy, it is more natural to consider isoentropic families of initial nuclei.

In order to define the initial density before scaling, we can use the constrained mean field method described above with a small constraint parameter $\lambda$ introduced to correctly treat the continuum $\left(\lambda=0.25 \mathrm{MeV} \mathrm{fm}^{-2}\right.$ in our simulation). The initial temperatures $T$ are defined in order to get the desired entropies $S$.

For numerical reasons, we have chosen to neglect the temperature dependence of the mean-field potential in the solution of the constrained mean-field. Therefore, the first stage of the initialization procedure is to find the static solution $\rho_{0}$ of the mean field problem at zero temperature with a small external constraint $\lambda r^{2}$. This provides us with a well defined one-body potential $U\left[\rho_{0}\right]$. Then, we have constructed a finite-temperature particle distribution as a hot gas of independent particles in this self-consistent potential, $U\left[\rho_{0}\right]$. Then using the scaling

$$
\vec{r} \longrightarrow \overrightarrow{r^{\prime}}=\alpha \vec{r}
$$




$$
\vec{p} \longrightarrow \overrightarrow{p^{\prime}}=\frac{\vec{p}}{\alpha}
$$

which conserves the entropy, we can generate various families of isoentropic initial conditions with different dilutions or compressions.

\subsection{Alternative approximate isothermal initialization}

If we do not take into account the self-consistent part of the mean-field potential, we see that energies scale approximately as

$$
\varepsilon \longrightarrow \varepsilon^{\prime} \simeq \varepsilon / \alpha^{2},
$$

showing that the temperatures approximately depend on $\alpha$ as

$$
T \longrightarrow T^{\prime} \simeq T / \alpha^{2}
$$

Therefore we can also generate a family of approximately isothermal initial densities by keeping $T^{\prime}$ constant, i.e. changing the temperature $T$ prior to the scaling. Results obtained with this approximation are in all points comparable to Constrained Hartree-Fock (CHF) simulations for a large range of initial conditions within the limitations of CHF discussed above. This gives good confidence in the scaling method and in the robustness of the conclusions we draw. Moreover, we would like to stress that, in fact, any initial conditions can be studied since the most important point is to use identical protocols in quantum and classical simulations so that the comparison is meaningful.

\section{Quantum model}

Following [13], we consider spherically symmetric nuclei. The one-body wave functions can be separated into their radial part, angular part and spinisospin part

$$
\Phi_{\alpha}(\vec{r}, s, t)=\frac{R_{n l}(r)}{r} Y_{l m}(\theta, \varphi) \chi_{\sigma, \tau}(s, t)
$$

in this expression $\vec{r}=(r, \theta, \varphi), \chi_{\sigma \tau}$ represents the spin and isospin part. In our special case, we consider that our system is spin and isospin saturated. Finally, $\alpha$ is an abbreviation for all the quantum number $(n, l, m, \sigma, \tau)$. 
For the considered spherical system, the TDHF equation reduces to a radial equation for the single particle wave functions

$$
i \hbar \frac{\partial R_{n l}(r, t)}{\partial t}=\left\{-\frac{\hbar^{2}}{2 m} \frac{\partial^{2}}{\partial r^{2}}+\frac{\hbar^{2} l(l+1)}{2 m r^{2}}+U[\rho](r, t)+V_{\text {Coulomb }}\right\} R_{n l}(r, t),
$$

In this equation $\hat{V}_{\text {coulomb }}$ is the Coulomb potentialf while $U$ is the densitydependent mean-field potential

$$
\hat{U}[\rho](r, t)=\frac{3}{4} t_{0} \rho(r, t)+\frac{2+\sigma}{16} \rho^{1+\sigma}(r, t),
$$

In this expression, $\rho$ is the local density

$$
\rho(r, t)=4 \sum_{n, l}(2 l+1) n_{n l} \frac{\left|R_{n l}(r, t)\right|^{2}}{4 \pi r^{2}},
$$

where $n_{n l}$ are the time-independent occupation numbers which only depend upon $n$ and $l$ quantum numbers. The factor 4 is coming from the spin and isospin degeneracy.

\subsection{Quantum initialization}

As discussed in the introduction, we have studied different types of initial conditions consisting in varying the dilution, the temperature and the entropy of the considered nuclei.

\subsubsection{Constrained Hartree-Fock}

In the constrained Hartree-Fock approach, the occupation numbers $n_{n l}$ are distributed according to the Fermi-Dirac distribution

$$
n_{n l}(T)=\frac{1}{\exp \left(\frac{\varepsilon_{n l}-\mu}{T}\right)+1}
$$

where the energies $\varepsilon_{n l}$ are the eigenvalue of the constrained Hamiltonian $h+$ $\lambda r^{2}$ while the single particle wave functions are nothing but the eigenstates of the constrained Hamiltonian. The parameters $\lambda$ and $\mu$ are defined requiring a given dilution and a given number of particles.

\footnotetext{
${ }^{2}$ In this calculation the coulomb field is considered in an approximate way by giving the charge $+1 / 2$ to each nucleons.
} 


\subsubsection{Scaling initialization}

In the second method, we use the energy levels and the wave functions of the zero temperature mean field in presence of a small external field $\left(\lambda r^{2}\right.$, $\lambda=0.25 \mathrm{MeV} \mathrm{fm}^{-2}$ ). This gives the energies $\varepsilon_{n l}$ and the associated single particle wave functions $R_{n l}$. Then, these states are occupied according to the Fermi-Dirac distribution (11). The temperature, $T$, is computed in order to get the desired entropy

$$
S=-4 \sum_{n, l}(2 l+1)\left(n_{n l} \log n_{n l}+\left(1-n_{n l}\right) \log \left(1-n_{n l}\right)\right)
$$

Finally, in order to get the appropriate dilution, the wave functions are rescaled by the transformation

$$
R_{n l}^{\prime}\left(r^{\prime}\right)=\frac{R_{n l}\left(\frac{r^{\prime}}{\alpha}\right)}{\alpha^{3 / 2}},
$$

This initialization procedure can also be used in order to generate scaled density at approximately constant temperature by scaling the temperature $T$ according to Eq. (6).

\subsection{Numerical details}

In practice, the self-consistent solution of the static Hartree Fock (at finite temperature and with a constraint $\lambda r^{2}$ ) has been found through an iterative application of the operator $1 /\left(\varepsilon_{n l}-\left(\hat{h}+\lambda \hat{r}^{2}\right)\right)$ to the single particle states $\Phi_{n l}$. In this procedure, at each step the one-body density is computed using the occupation numbers (11) where the energy is given by $\varepsilon_{n l}=\left\langle\Phi_{n l}\left|\hat{h}+\lambda \hat{r}^{2}\right| \Phi_{n l}\right\rangle$. We start with the oscillator wave functions and after many iterations, we converge to the solution of our self-consistent field. We have controlled that this method gives same results as an iterative diagonalization of $\hat{h}+\lambda \hat{r}^{2}$. From a practical point of view, we have done the simulation with up to 40 orbitals. We have chosen a step of $\Delta r=0.2 \mathrm{fm}$ in r-space.

After having initialized the system, we let it evolve according to the TDHF equation written in $r$-space(8). During the expansion of the system, occupation numbers are kept fixed, implying the conservation of the entropy of the total system. Note that, in the following, we will sometime study only 
part of the system; for such a sub-system the evolution will not be isoentropic anymore.

We have performed simulations on a lattice of size $480 \mathrm{fm}$, this size is big enough to avoid bouncing of particle against the wall. For the evolution, we have taken a step in time of $0.75 \mathrm{fm} / \mathrm{c}$. Before presenting results, we will present in an analogous manner the method we have used for the semiclassical simulation.

\section{Semi-classical model}

The equivalent semi-classical equations are obtained by taking the first term of the $\hbar$ expansion of Wigner transform of the TDHF equation. This leads to the Vlasov equation

$$
\frac{\partial f(\vec{r}, \vec{p}, t)}{\partial t}=\{h[f], f\}
$$

where $\{.,$.$\} are the usual Poisson brackets and f(\vec{r}, \vec{p}, t)$ and $h[f]$ are respectively the one-body phase-space density and the associated self-consistent mean-field. One of the methods widely used for solving the Vlasov equation is the lattice Hamiltonian method developed in ref. 116.

In this method, the r-space is discretized into a three-dimensional cubic lattice. Each nucleon is represented by a number $N_{\text {test }}$ of "test particles". The density is calculated on each site of the lattice using the following algorithm: considering a site "i", each particle contained in the neighboring cubes participates to the density at the point "i" with a given weight which depends on the distance between the particle and the considered site. Formally, this is obtained through the convolution

$$
\bar{\rho}=\rho \otimes G
$$

where the unfold density is simply given by the density of point-like testparticles

$$
\rho\left(x_{i}, y_{i}, z_{i}\right)=\frac{1}{N_{\text {test }}} \sum_{n=1}^{A N_{\text {test }}} \delta\left(x_{i}-x_{n}\right) \delta\left(y_{i}-y_{n}\right) \delta\left(z_{i}-z_{n}\right)
$$

and where the weight function is given by a distribution of the density over $2 m$ neighboring sites. 


$$
\begin{aligned}
G(x, y, z) & =g(x) g(y) g(z) \\
g(\alpha) & =\frac{(m \Delta r-|\alpha|)}{(m \Delta r)^{2}} \Theta(m \Delta r-|\alpha|)
\end{aligned}
$$

In this expression, $\Theta$ corresponds to the Heavyside function, so that $(m \Delta r)$ corresponds to the range of the smoothing used to compute the density. Finally we get the following density at each site $i$ of the considered lattice

$$
\bar{\rho}\left(x_{i}, y_{i}, z_{i}\right)=\frac{1}{N_{\text {test }}} \sum_{n=1}^{A N_{\text {test }}} g\left(x_{i}-x_{n}\right) g\left(y_{i}-y_{n}\right) g\left(z_{i}-z_{n}\right)
$$

This density is used to compute the energy functional $E[\bar{\rho}]$ (see eq. (2)) at each point $i$ of the lattice, in order to define the Hamiltonian $\mathcal{H}$ of the system [16]

$$
\mathcal{H}\left(\vec{r}_{n}, \vec{p}_{n}\right)=\sum_{n} \frac{p_{n}^{2}}{2 m}+\sum_{i} \Delta r^{3} E[\bar{\rho}]\left(\vec{r}_{i}\right)+E_{\text {Coulomb }}
$$

where, following our conventions, the sum over $n$ runs over all the testparticles while the sum over i corresponds to the sum over lattice sites. The evolution of the system consists in the classical Hamilton equations for each test particle $n$ which have been solved using the standard leap-frog algorithm

$$
\begin{aligned}
\vec{r}(t+\Delta t) & =\vec{r}(t-\Delta t)+2 \Delta t \frac{\vec{p}(t)}{m}, \\
\vec{p}(t+2 \Delta t) & =\vec{p}(t)+2 \Delta t \vec{F}(t+\Delta t),
\end{aligned}
$$

where the force is calculated according to

$$
\vec{F}=-\frac{\partial \mathcal{H}\left(\vec{r}_{n}, \vec{p}_{n}\right)}{\partial \vec{r}_{n}}
$$

Using the expression (20) for the Hamiltonian $\mathcal{H}$, it is easy to demonstrate that the force $\vec{F}$ derives from a potential $\bar{U}$ which is related to the mean field potential $U[\bar{\rho}]$ through the relation

$$
\bar{U}(x, y, z)=\frac{1}{(2 m)^{3}} \sum_{i} U\left(x_{i}, y_{i}, z_{i}\right)
$$

where the sum runs over the $2 m$ neighboring cells of the position $(x, y, z)$. 
This method has demonstrated its accuracy and is now widely used in theoretical description of Heavy-Ion collision [16. We have thus used it and compared results with quantum simulation. It should be noticed that other methods are reported in the literature using either slightly different lattice discretization of the density or gaussian smoothing in order to fold the pointlike test-particle density into a smooth nucleon density. Therefore, all the semi-classical methods contain a smoothing procedure in order to generate the nucleon density used in the energy functional. The presented results do not depend on the particular numerical implementation of this smoothing.

In practice, we have adopted the value $N_{\text {test }}=200$ which corresponds to a rather large number in such type of simulations. For the Vlasov evolution, we have taken the three dimensional code TWINGO developed in ref. [19, 18]. The simulation are done with a range for the convoluting function $\mathrm{g}$ of $2 \mathrm{fm}$, the lattice grid has a step of $1 \mathrm{fm}$ and the step in time is the same as in the quantum simulation: $0.75 \mathrm{fm} / \mathrm{c}$. The total $\mathrm{r}$-space is a box of size $40 \times 40 \times 40$, when a particle reachs the boundary, it is not evolved anymore.

Before describing the results, we will get more insight into the simulation and in particular into the initialization procedure.

\subsection{Initialization and stable states of the Vlasov dy- namics.}

\subsubsection{Constrained Thomas-Fermi solution}

The first point is to define stable spherical nucleus at temperature $T$ under

the constraint $\lambda r^{2}$. For this purpose, we have only to compute the spherical one-body phase space density $f(r, p)$ of the Thomas-Fermi type

$$
f(\vec{r}, \vec{p}, T)=\left(\exp \left(\frac{\vec{p}^{2} / 2 m+\bar{U}(\vec{r})+\lambda r^{2}+V_{\text {Coulomb }}-\mu}{T}\right)+1\right)^{-1}
$$

which indeed maximizes the entropy

$$
S=4 \int \cdots \int \frac{d^{3} r d^{3} p}{h^{3}}(f \log f+(1-f) \log (1-f))
$$

under the various constraints. In expression (25), $\mu$ is the chemical potential and $\bar{U}(\vec{r})$ is the potential consistent with the lattice Hamiltonian method (see Eq. (24) )which is obtained using the folded density $\bar{\rho}$. Note that, in order to 
speed up the initialization process, we have used the spherical symmetry: we have replaced Eqs. (19) and (24) by convolutions with a spherical function

$$
\begin{aligned}
\bar{\rho}(r) & =\int_{r=0}^{r^{\prime}=+\infty} \rho\left(r^{\prime}\right) g\left(r^{\prime}-r\right) 4 \pi r^{\prime 2} d r^{\prime} \\
\bar{U}(r) & =\int_{r=0}^{r^{\prime}=+\infty} U[\bar{\rho}]\left(r^{\prime}\right) g\left(r^{\prime}-r\right) 4 \pi r^{\prime 2} d r^{\prime}
\end{aligned}
$$

where the function $g$ is a function analogous to the expression (18)

$$
g(r)=\frac{\left(r_{0}-r\right)}{\left(r_{0}\right)^{2}} \Theta\left(r_{0}-r\right)
$$

but with a range $r_{0}$ adapted to provide the same averaged squared-radius as $G$.

The numerical procedure used to build a constrained Thomas-Fermi distribution in phase space can be separated in two steps:

- the first one consists in an iterative definition of $f(r, p, T)$ : starting with a potential (at the first iteration the potential is assumed to be a WoodSaxon), we calculate the phase space density according to expression (25) using a chemical potential $\mu$ which conserves the particle number. Then, we obtain the density $\rho(r)$ by integrating $f(r, p, T)$ over $p$ and we calculate the folded density $\bar{\rho}(r)$ and the associated potential $\bar{U}(r)$ using Fast Fourier transform techniques. Finally, we iterate the operation until a full convergence is reached. This provides us with a continuous self-consistent phase-space density $f(r, p, T)$.

- The second step consists in distributing test particles according to the phase space distribution, $f(r, p, T)$ using a standard Metropolis algorithm [17].

The stability over time of a nucleus initialized in its ground state demonstrates the accuracy of this method in finding stable solutions in lattice Hamiltonian framework. p

\footnotetext{
${ }^{3}$ Indeed, we have not observed the emission of a single test-particle during the typical simulation time used in the following.(more than $300 \mathrm{fm} / \mathrm{c}$ )
} 


\subsubsection{Scaling procedure}

As already discussed in the quantum case, we have also studied a second class of initial conditions namely those obtained through a scaling. In order to follow the approximation made in the quantum simulation, the meanfield potential, included in the definition of the finite temperature phase space density (eq. (25)), has been approximated by the zero-temperature one (including a small constraining external potential $\lambda r^{2}, \lambda=0.25 \mathrm{MeV} \mathrm{fm}^{-2}$ ). For each of these initial conditions, the temperature is chosen in order to get the expected entropy (26).Then, the test-particle distribution is generated using the Metropolis algorithm as discussed above. Finally the momenta and the positions of the test particles are scaled according to eq. (3). Since this scaling preserves $f$ and the phase space volume $d^{3} r d^{3} p / h^{3}$, it does not change the entropy (26). We are thus generating a family of isoentropic initial conditions.

This scaling procedure can be also used to construct an ensemble of initial conditions which can be considered as isothermal by changing the temperature $T$ in order to compensate the effects of the dilution (or the compression) on the temperature prior to the scaling according to Eq. (6).

\subsection{Discussion on smoothing in semi-classical methods}

In this section, we will discuss the possible bias of the comparison between quantum and semi-classical simulations due to the test-particle method. Indeed, in any semi-classical approach based on a test-particle method, a smoothing of the phase space density should be included in order to compute the mean-field potential. As already mentioned in [25], this smoothing

acts as an effective range for the forces. Indeed, considering the density of particles $\rho$, the actual numerical smoothed density $\hat{\rho}$ is given by the folding product with a smooth function $g$

$$
\hat{\rho}=\rho \otimes g
$$

The function $g$ used in our simulations is given by equation (16) but $g$ could take other expression. Expending the Fourier transform of $g$ at the second order in $k$ this folding product can be approximated by

$$
\hat{\rho}=\rho-\beta \Delta \rho
$$


where $\beta$ is the range of the function $g$. Then, the time-dependent mean-field becomes

$$
U[\hat{\rho}]=\frac{3}{4} t_{0} \rho+\frac{(2+\sigma)}{16} t_{3} \rho^{1+\sigma}-\beta \Delta \rho\left(\frac{3}{4} t_{0}+\frac{(2+\sigma)(1+\sigma)}{16} t_{3} \rho^{\sigma}\right)
$$

where one can recognize a typical finite range term in the last part of Eq. (32). However, it should be noticed that the above expression corresponds to a very specific surface contribution.

As a direct consequence, due to this additional surface energy, we will see that energies calculated in semi-classical approximations will be greater that those calculated in quantum simulations. This might seems surprising since in absence of quantum correction terms in the semi-classical potential, one would expect the surface energy present in the semi-classical picture to be smaller than the quantum equivalent (or even zero). In fact, this surface energy is only generated by the numerical method. Indeed, in the quantum case, since wave functions are not localized, no smoothing is needed and the algorithm does not introduce spurious additional surface term. However, it is well known that even with a zero range force with no momentum dependence (to simulate a finite-range), a quantum calculation will exhibit non trivial surface properties due to the role of the kinetic term and because of the self consistence effects on the wave functions. Conversely, a semiclassical calculation with a zero range force should present sharp edges (at zero temperature). Then, surface properties, in semi-classical calculation, are influenced by the smoothing procedure.

This difference in the surface properties can be seen as a major drawback of semi-classical approaches. Of course one might think to take advantage of the presence of this numerical finite range in semi-classical pictures in order to mimics some quantum effects or some surface terms in the considered interaction. In the next chapter, we will more carefully investigate this possibility, however, we can already mention some difficulties:

- It is never easy to rely on the numerics in order to mimic physical effects.

- One might try to select the numerical coarse-graining, but normally, it is fixed by some other arguments such as numerical accuracy (see Eq. (32)). 
- since the surface effects are coming from the folding procedure in the mean-field potential their importance is fixed by the mean field strength (see Eq. (32)). Since this strength is density dependent, the surface effect will have the same dependence. This might be not realistic since they are supposed to mimic surface effects of various origin and so with various density dependence: interaction range, quantum kinetic term, finite range of wave functions and self-consistent effects. This point is partly discussed in ref. 23.

- In any realistic cases, where surface term exists in the interaction, one should be careful in order to not double count these finite size effects.

We observe that, a semi-classical simulation, which is supposed to be based on a given force (11), includes a spurious additional interaction (32). As we will see, this will contribute to the observed differences with the quantum simulation associated to (1). However, realistic interactions usually include a finite range term, so that it might be interesting to investigate the possibility to mimics quantum dynamics using a finite range interaction with a semiclassical approach eventually taking into account the numerical surface term in the potential.

\subsection{Discussion of surface effects.}

In this section we consider a mean-field potential taking into account a finite range effect in the TDHF mean-field

$$
U(\rho)=\frac{3}{4} t_{0} \rho+\frac{(\sigma+2)}{16} t_{3} \rho^{\sigma+1}+c \nabla^{2} \rho .
$$

Usually, in the TDHF calculation, the Laplacian terms is simulated by a finite range potential of the Yukawa type in order to avoid well-known numerical instabilities 24]. Therefore, the mean-field potential has been computed using the relation

$$
U(\rho)=\frac{3}{4} t_{0}^{\prime} \rho+\frac{(\sigma+2)}{16} t_{3} \rho^{\sigma+1}+V_{0} \rho \otimes Y .
$$

\footnotetext{
${ }^{4}$ In the skyrme force, the parameter $c$ is related to the parameters $t_{1}$ and $t_{2}$ 14, 24.
} 
where $Y$ is a Yukawa folding function

$$
Y(r)=\frac{\exp (-|r| / a)}{|r| / a}
$$

and where $t_{0}^{\prime}$ is function of $a, V_{0}$ and the $t_{0}$ used in the classical calculation. Indeed, expanding the Fourier transform of the folding product up to the second order in $k$ we can derive the following expression for the potential

$$
U(\rho)=\frac{3}{4} \underbrace{\left(t_{0}^{\prime}+\frac{16 \pi V_{0} a^{3}}{3}\right)}_{t_{0}} \rho+\frac{(\sigma+2)}{16} t_{3} \rho^{\sigma+1}+\underbrace{2 V_{0} \pi a^{5}}_{c} \nabla^{2} \rho .
$$

For numerical applications, we have chosen the same values of $t_{0}$ and $t_{3}$ as before in the semi-classical simulation. For the quantum case, we have chosen for $a$ and $V_{0}$ respectively $0.37 \mathrm{fm}$ and $-200 \mathrm{MeV}$ (which gives a $t_{0}^{\prime}$ value of $830.3 \mathrm{MeV} \mathrm{fm}^{3}$ ) in order to have almost the same energy for the ground state in quantum and in semi-classical case at zero temperature. In the following, this particular simulation will be referred as "Quantum+surface".

\section{Results}

In this section we will focus on a detailed comparison of the two considered approaches, the semi-classical Vlasov dynamics and the full quantum TDHF treatment (with and without additional surface terms). We will take a hot and compressed or diluted Double magic ${ }^{40} \mathrm{Ca}$ nucleus as a test case.

\subsection{Initialization of nuclei under extreme conditions}

In this section, we will illustrate different initial conditions of compression and temperature. We will first discuss density profiles obtained after the initialization step and then, present the various excited nuclei we are considering in the evolution.

\subsubsection{Density profiles}

In figure (11), we compare quantum and semi-classical constrained mean-field solutions obtained at various temperature $(T=0,5,10$ and $15 \mathrm{MeV})$ with a 
small constrained field $\lambda r^{2}$ with $\lambda=0.25 \mathrm{MeV} / \mathrm{fm}^{2}$. As expected, the semiclassical simulation, which, in addition to the smoothing procedure, does not include shell effects, presents smoother initial density profiles. Directly connected to the smoothing, the quantal density profiles always present a smoother surface than the semi-classical one. In addition, the Root Mean Square radii (RMS) and the central densities are reported in table 1. We observe that these quantities are slightly different in semi-classical case compared to both quantum simulations. In particular, the temperature dependence of such global observables indicates a different response of the nuclei to the heating process within the two approaches.

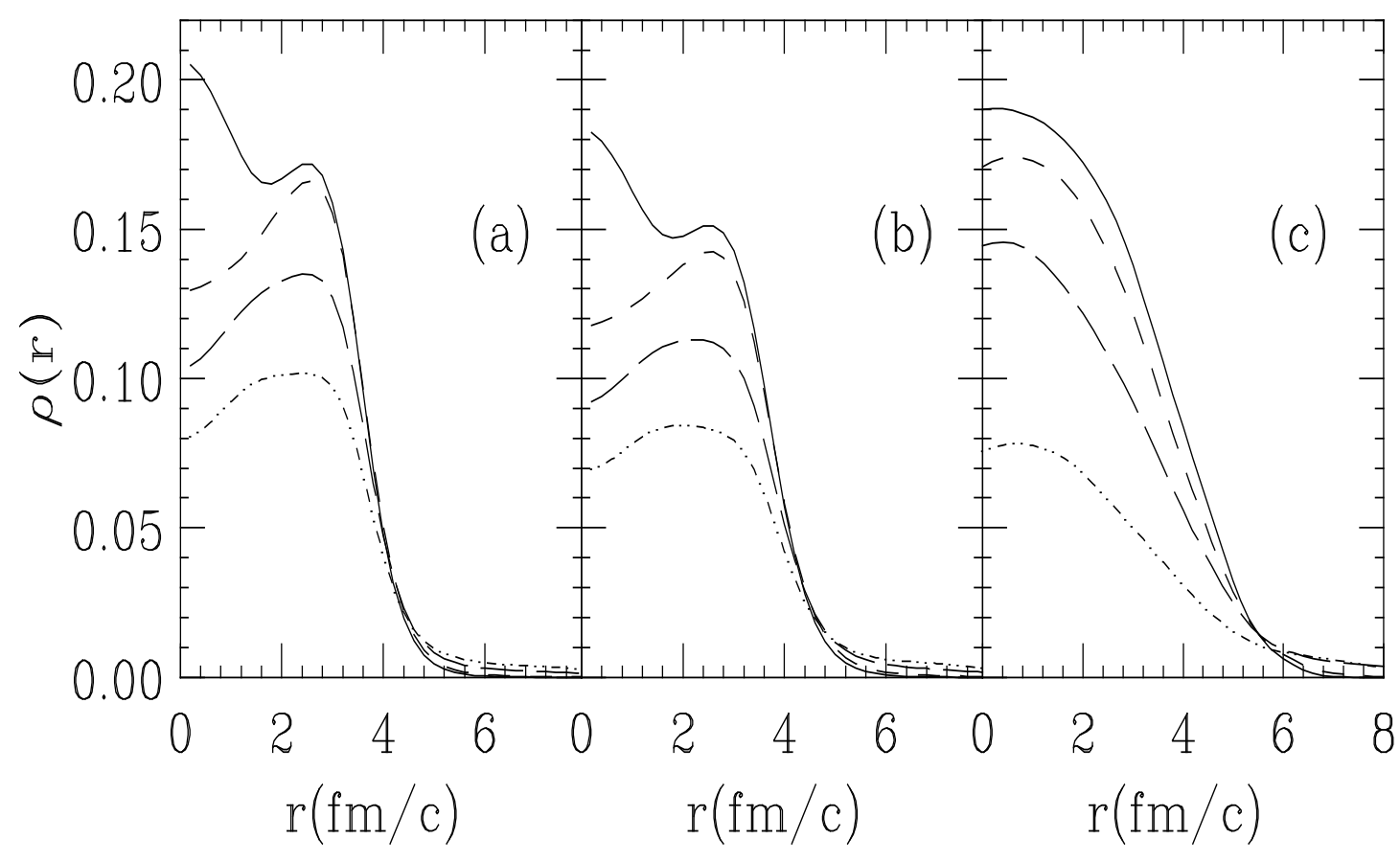

Figure 1: Initial density profiles of a ${ }^{40} \mathrm{Ca}$ nucleus at various temperatures for a constraining field $\lambda r^{2}$ with $\lambda=0.25 \mathrm{MeV} / \mathrm{fm}^{2}$ : a) Quantum approach; b) Quantum+surface c)Semi-classical. In each figures are displayed temperature $T=0 \mathrm{MeV}$ (solid line), $5 \mathrm{MeV}$ (dashed line), $10 \mathrm{MeV}$ (long-dashed line), $15 \mathrm{MeV}$ (short-dashed line). 


\begin{tabular}{|c|c|c|c|c|c|c|}
\hline \multirow{2}{*}{} & \multicolumn{2}{|c|}{ Quantum } & \multicolumn{2}{c|}{$\begin{array}{c}\text { Quantum } \\
\text { +Surface }\end{array}$} & \multicolumn{2}{c|}{ Classical } \\
\cline { 2 - 7 } & RMS & $\rho$ & RMS & $\rho$ & RMS & $\rho$ \\
\hline \hline$T=0 M e V$ & 3.14 & 0.172 & 3.31 & 0.153 & 3.09 & 0.196 \\
\hline$T=5 \mathrm{MeV}$ & 3.30 & 0.148 & 3.53 & 0.132 & 3.36 & 0.179 \\
\hline$T=10 \mathrm{MeV}$ & 4.30 & 0.130 & 4.65 & 0.109 & 4.93 & 0.144 \\
\hline$T=15 \mathrm{MeV}$ & 5.55 & 0.098 & 5.94 & 0.082 & 8.03 & 0.080 \\
\hline
\end{tabular}

Table 1: Root Mean Square radius (RMS) and central densities of a ${ }^{40} \mathrm{Ca}$ nucleus initialized with a constraining field $\lambda r^{2}$ with $\lambda=0.25 \mathrm{MeV} / \mathrm{fm}^{2}$ at various temperatures

In the scaling procedure, for isoentropic initial conditions, density profiles of compressed or dilated nuclei will be obtained by a scaling of those displayed in Fig.1.

\subsubsection{Isoentropic initial conditions}

Important information about a system is provided by the total energy $E$ as a function of $\rho$ for various entropies. When considering infinite nuclear matter this is the usual Equation Of state (EOS), $E=E(\rho, S)$ as shown in fig. 2 for the considered interaction. This EOS was extensively used in order to understand the properties and the dynamics of nuclear matter. For each entropy, the minimum of the energy corresponds to a stationary isoentropic solution. Furthermore, the second derivative of the energy with respect to $\rho$ around the stationary point is directly related to the frequency of the monopole oscillation around this point. The isoentropic spinodal region is defined as points where this curvature is negative or equal to zero. This region is of particular interest since one of the possible scenarii of multifragmentation is that a highly excited nucleus could enter this region and become unstable against the partition of the matter in fragments.

Let us built an equivalent of the EOS for a finite system. In order to do so, we can plot the relation between the central density $\rho$ and the energy per particles $E / A$ of the system for the various initial entropies considered. In Fig.3, we report four different curves which correspond to entropies $S / k_{B}=$ $0,1.10,2.35$ and 3.28. These curves are obtained by applying the general scaling procedure to each models: occupations numbers are kept fixed while different compressions/dilations are considered. 


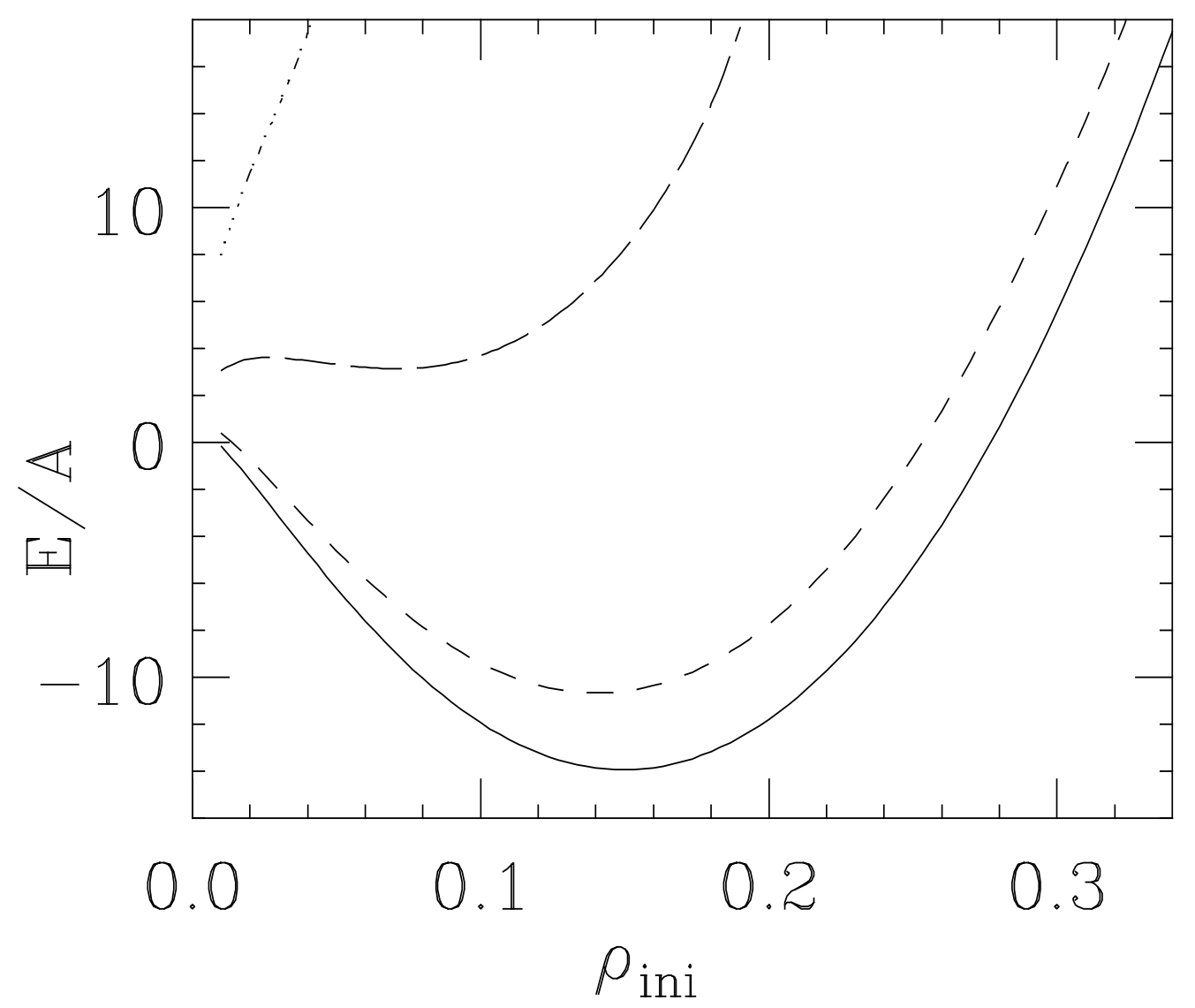

Figure 2: Binding energy of the infinite nuclear matter as a function of its density, the curves correspond respectively to $S / k_{B}=0,1.1,2.35$ and 3.28. 


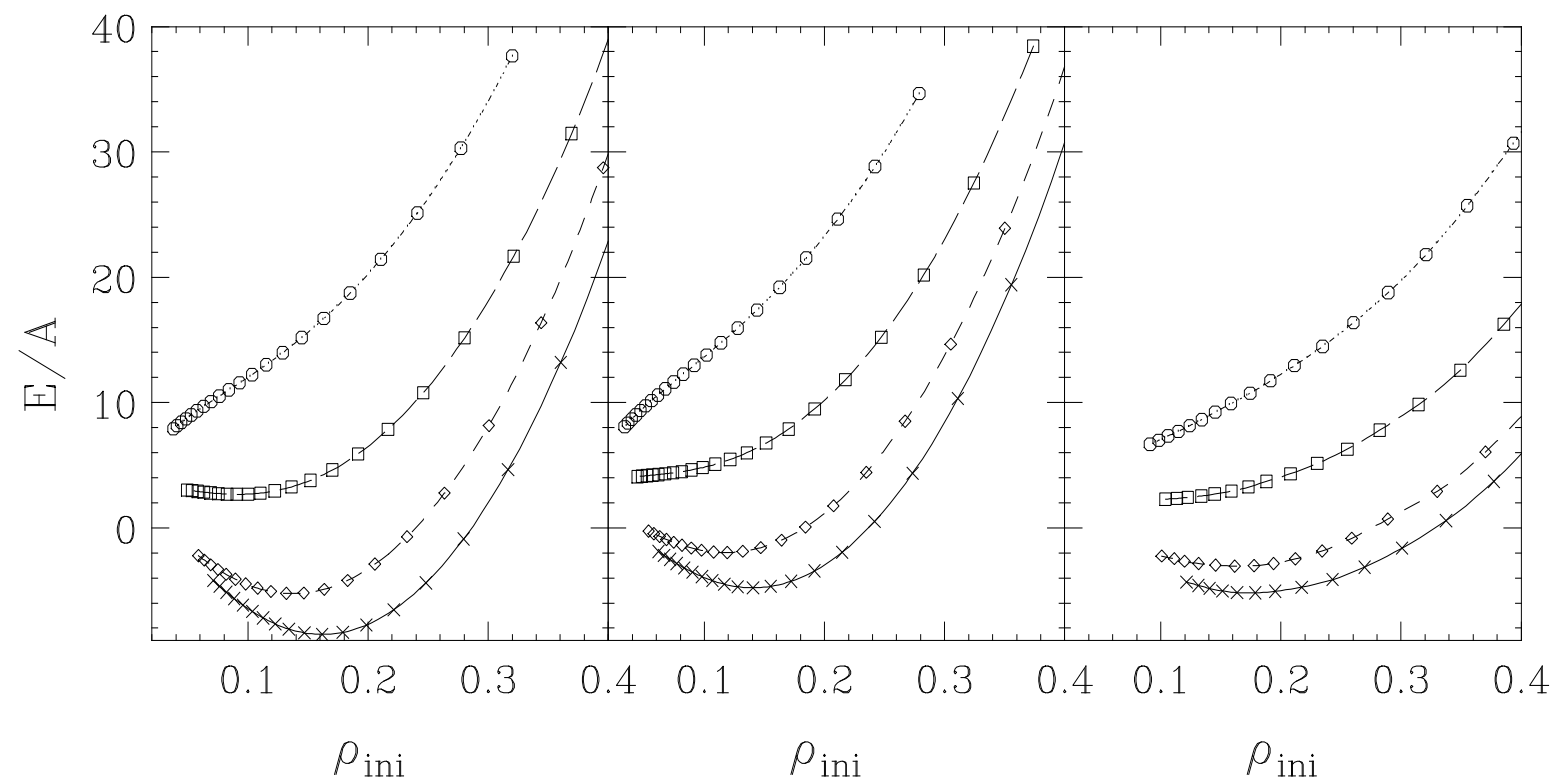

Figure 3: Binding energy of a hot and compressed/diluted Ca nucleus as a function of its central density, the curves corresponds respectively from bottom to top to $S / k_{B}=0(\times), 1.1(\diamond), 2.35(\square)$ and $3.28(\circ)$ on each figures. Left: Quantum (without surface). Middle: Quantum+Surface. Right: Semiclassical. 
Looking first at the minimum of the curve $S / k_{B}=0$, we see that the semi-classical stationary point has an energy greater than that of the quantum system (without surface).This is a direct consequence of the smoothing procedure. The additional surface term gives an extra energy of the order of $3 \mathrm{MeV}$. Note that, in the second quantal calculation (middle part), the surface term was adjusted in order to have the same ground-state energy as in the semi-classical case. If we now focus on the curvature of isoentropic curves, we see that they appear smaller in semi-classical than in both quantal calculations. Note that, if we suppose an evolution at contant entropy and energy, these curvatures are direcly related to the response to a monopolar excitation. In such a context, according to figure 3 , the semi-classical model should have a larger amplitude than quantal one. However, in dynamical simulations, physical systems have neither constant energy nor constant entropy in particular due to particle evaporation. As we will show later, the difference in evaporation processes in addition to the damping due to the use of test-particle method will strongly reduce the amplitude of monopolar vibrations in semi-classical simulations making it smaller than the quantum analogue.

Finally, we want to point out that temperature creates more dilution and disorder in semi-classical calculation than in quantum one as we already noticed in Fig. 1. We can quantify this more easily considering for instance the dependence of the entropy with temperature at a fixed compression factor. In Fig. $\mathbb{\text { t }}$, we report such a dependence for the three models when no compression is applied. We see in particular, that higher temperatures are necessary in quantal cases in order to create the same entropy than in a semi-classical simulation. This is a great difference between quantum and classical treatment of nuclei.

\subsection{Monopole oscillations and thermal expansion}

\subsubsection{Examples of expansion}

Let us now consider the time evolution of the density profiles presented in figure 10 for the constrained method. In figure 5, we present the evolution of a hot system for different temperature $T=5,10,15 \mathrm{MeV}$. From a qualitative point of view, the semi-classical and the TDHF evolutions are in reasonable agreement except at high temperature, for which, the semiclassical nucleus does not resist to the heat and to vaporizes easily than 


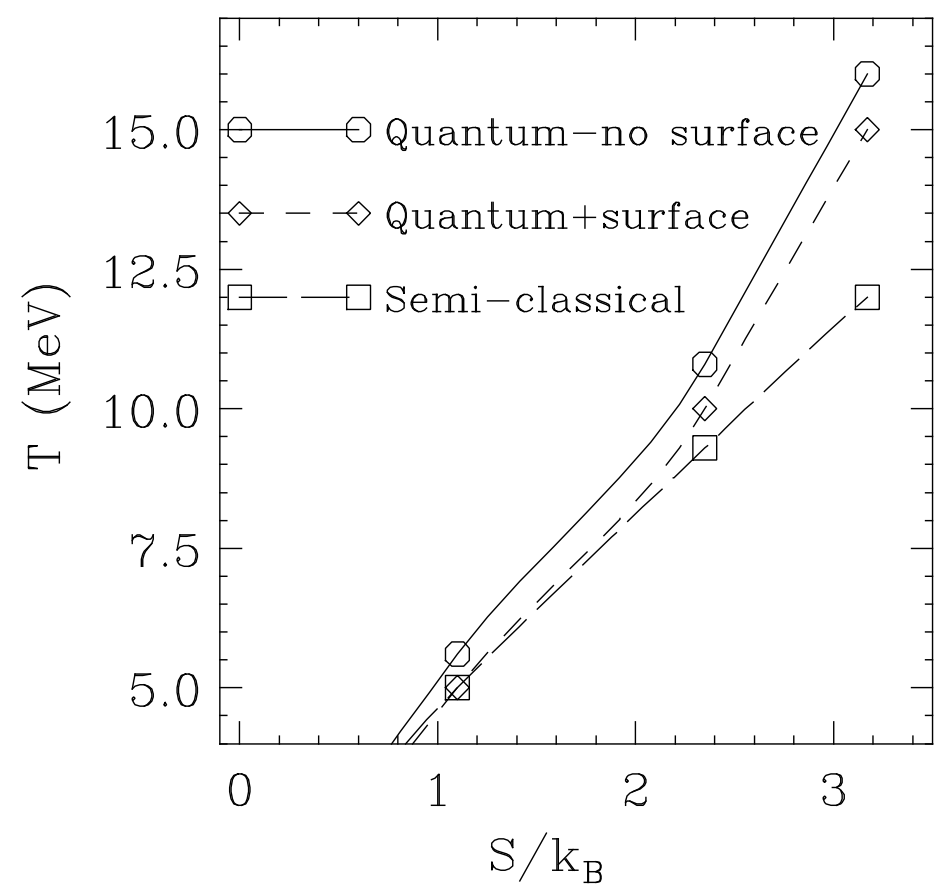

Figure 4: Entropy as a function of the temperature for a ${ }^{40} \mathrm{Ca}$ nuclei obtained when a small external field $\lambda r^{2}$ with $\lambda=0.25 \mathrm{MeV} / \mathrm{fm}^{2}$ is applied. Solid line: Quantum (without surface), dashed line: Quantum+surface, long-dashed line: Semi-classical. 
its quantum analogue. In fact, not only the vaporization process appears different and seems to start at a lower value of the initial temperature but also the whole particle evaporation is faster.

Before investigating in more details this observation and before discussing the possible reasons of this behavior let us first study quantitatively the various observed differences between the semi-classical and the quantum approaches.

\subsubsection{Illustration of the monopole oscillation}

Let us first study the collective monopole motion associated with a compressed or diluted system.

Figure 6 shows the central density as a function of time, predicted by the quantum evolution for a heated and compressed $\mathrm{Ca}$ nucleus initialized at $T=5 \mathrm{MeV}$ and with an initial density $\rho_{i n i}=0.23 \mathrm{fm}^{-3}$. In all cases, the central density presents an oscillating pattern due to the monopole vibration of the nucleus. In addition, in Quantum simulation beatings of different modes which are characteristic of the Landau spreading of the breathing mode onto various components [21, 22], are observed even at high temperature (see Fig. 6 (Top)). Note also, that the central density in semi-classical simulations, exhibits an important noise added on top to the monopole vibration. These fluctuations are due to the finite number of test-particles. In order to quantitatively study this vibration, for different initial conditions, we have considered two observables as a function of the initial entropy and density:

- the value of the minimum of the central density reached during the evolution (noted $\rho_{\min }$ which provides a measure of the amplitude of the monopole vibration;

- the time needed to reach this minimum (noted $t_{\min }$ )which, in case of a pure oscillatory motion, is directly related to half of the period of the vibration.

In the following, we will present results where events are grouped according to their entropies. 


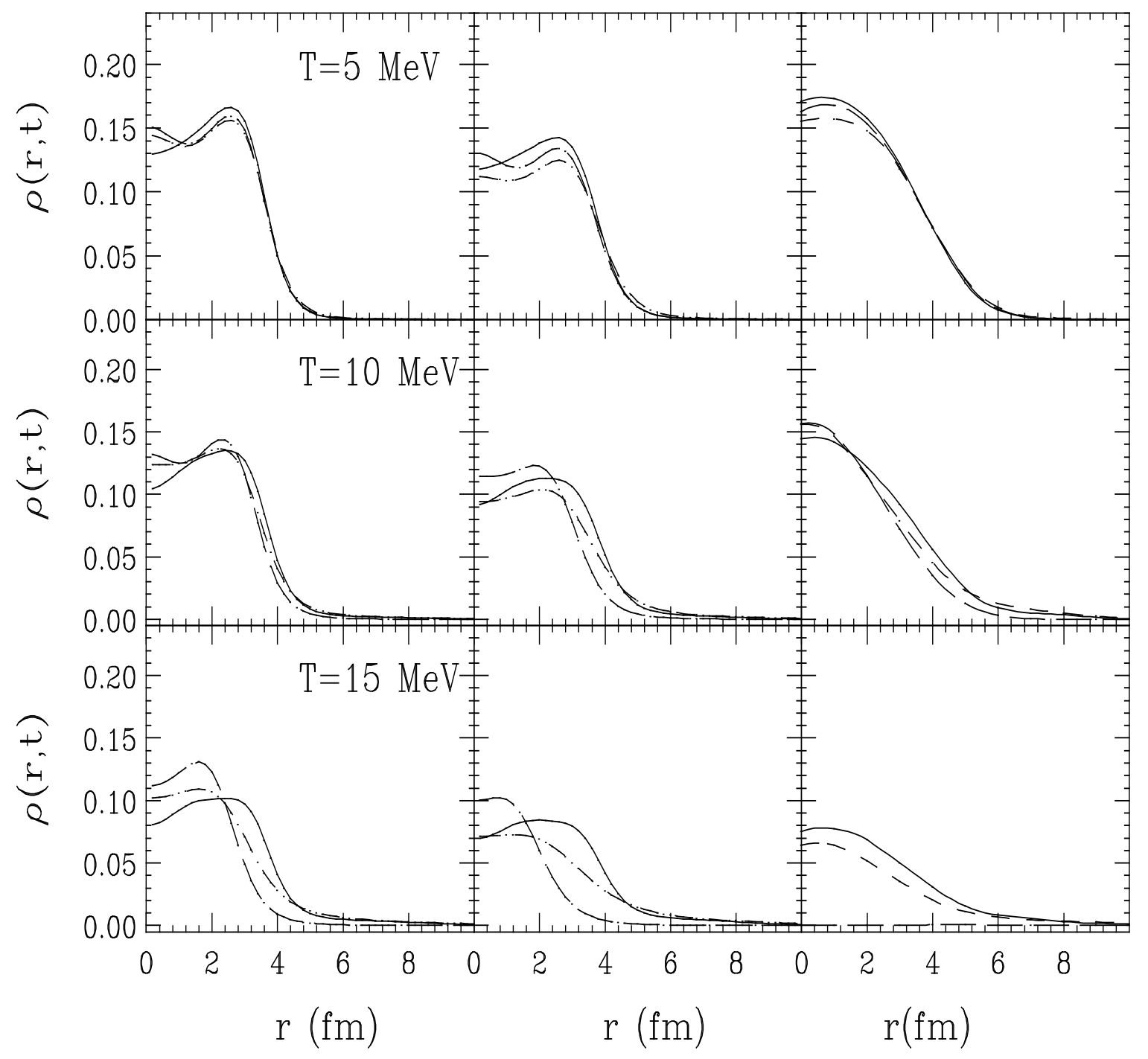

Figure 5: Density profiles for various initial temperatures - for $T=5,10,15$ $\mathrm{MeV}$ (from the top to the bottom) - and for: Left part: Quantum (Without surface). Middle Part: Quantum+Surface. Right part: Semi-classical. In each figure various curves corresponds to different times: Solid line: $t=0$ $\mathrm{fm} / \mathrm{c}$. Dashed line: $t=30 \mathrm{fm} / \mathrm{c}$. Long-dashed line: $t=300 \mathrm{fm} / \mathrm{c}$. 


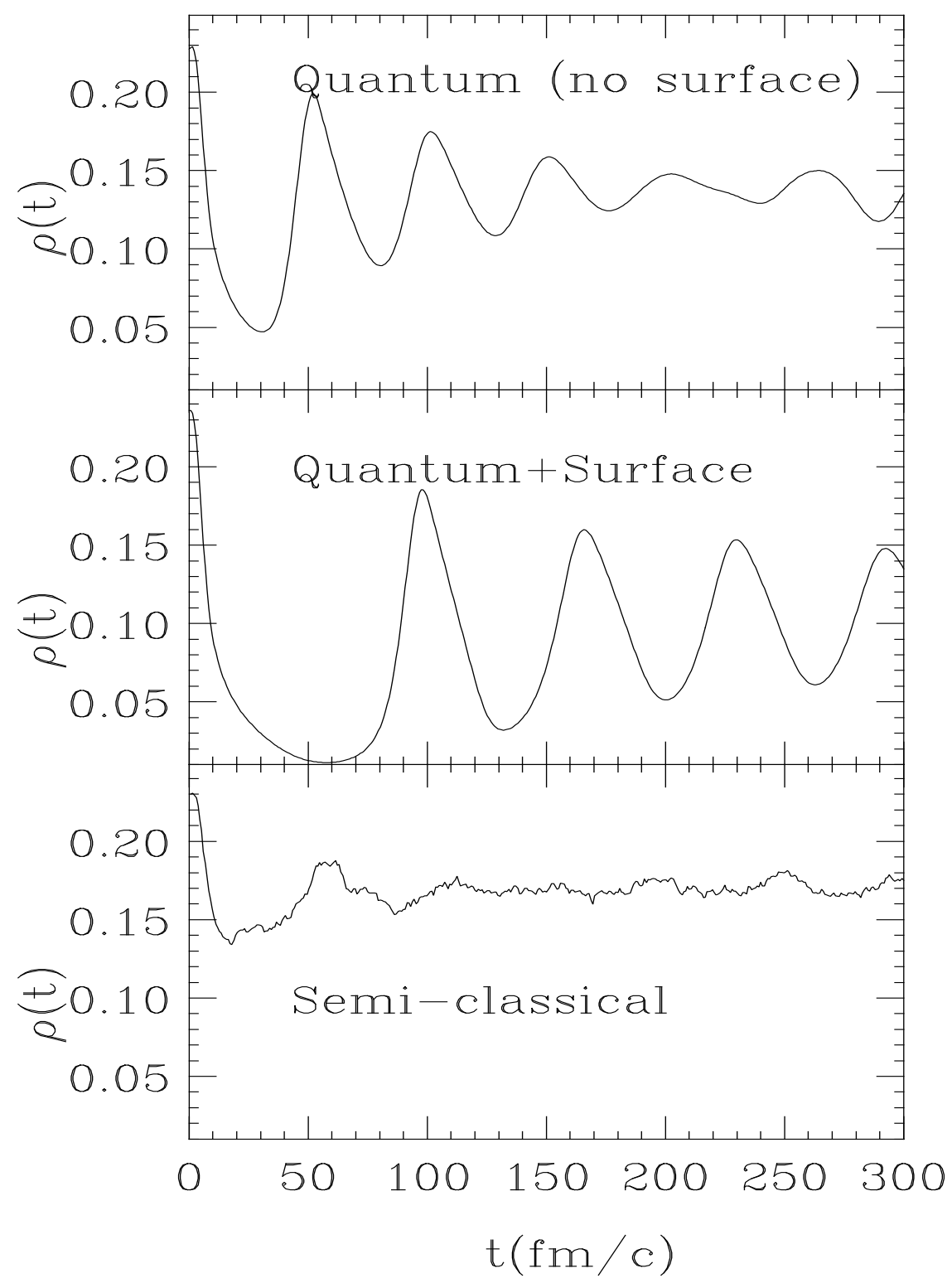

Figure 6: Illustration of oscillation of the central density in time for different models. In all models, initial conditions correspond to a ${ }^{40} \mathrm{Ca}$ nuclei at $T=5$ $\mathrm{MeV}$ and an initial density $\rho_{i n i}=0.23 \mathrm{fm}^{-3}$. Top: Quantum (Without surface). Middle: Quantum+surface. Bottom: Semi-classical. 


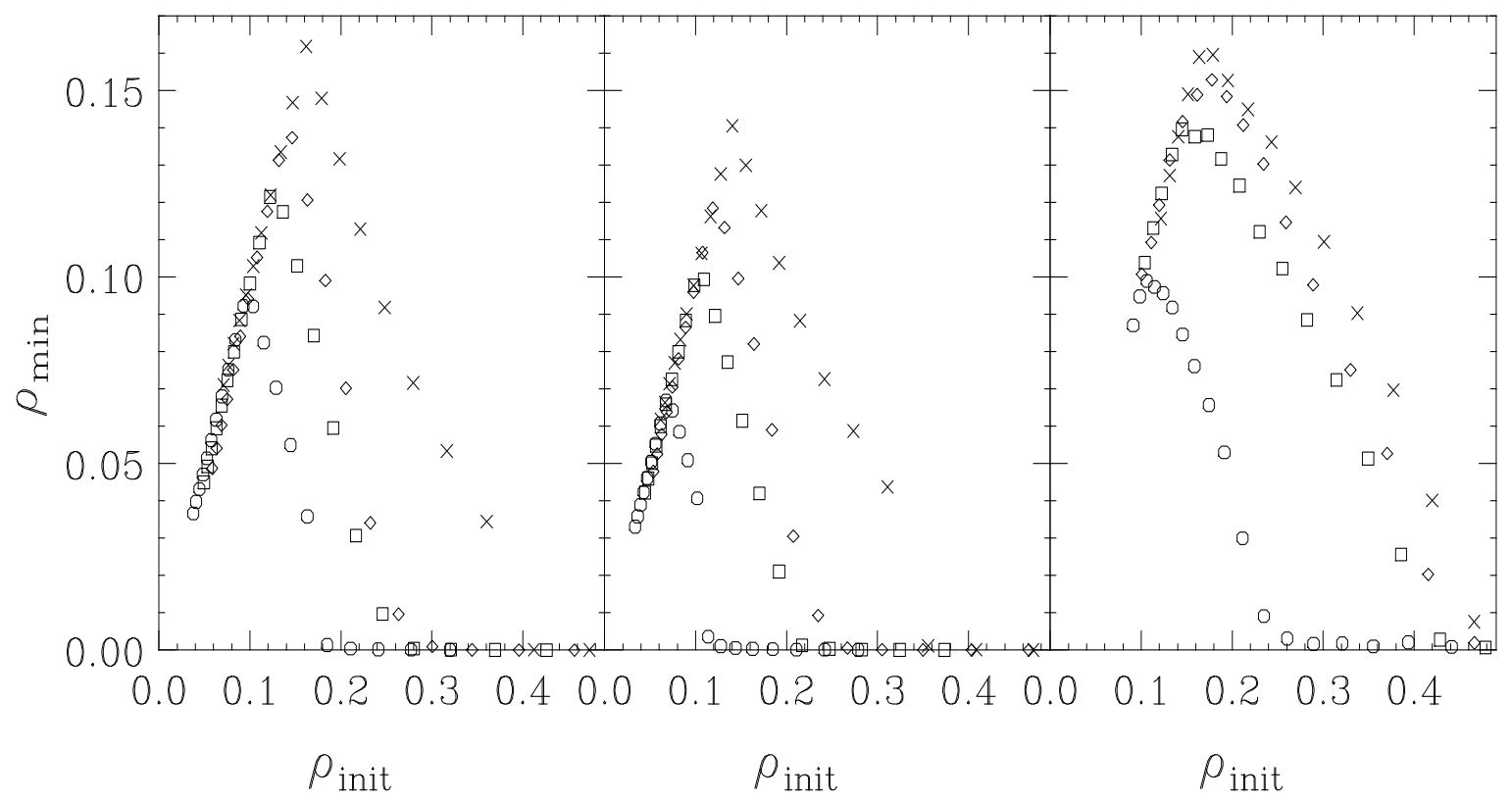

Figure 7: The central density at the maximum of the dilution (turning point) as a function of the initial density for a ${ }^{40} \mathrm{Ca}$ nucleus at various initial temperatures (from top to bottom points corresponds respectively to $S / k_{B}=0(\times), 1.1(\diamond), 2.35(\square)$ and $\left.3.28(\circ)\right)$. Left: Quantum (without surface). Middle: Quantum+Surface. Right: Semi-classical simulation. 


\subsubsection{Expansion of isoentropic initial conditions}

The value of the first minimum of the central density oscillations $\left(\rho_{\min }\right)$ is displayed in figure (7) as a function of the value of the initial density $\left(\rho_{\text {init }}\right)$. For a given entropy, this curve presents two branches. The first one (low initial density region) corresponds to the case of a minimum density equal to the initial one, i.e. the nucleus is already diluted compared to the stationary initial condition. Therefore this first branch corresponds to $\rho_{\min }=\rho_{\text {init }}$. The second branch gives the amplitude of the collective motion, i.e. the maximum dilution, as a function of the initial compression. The point where these two branches met is the stationary point for which no oscillations are observed. Note also that at finite temperature even if the system is stationary, it cools down by particle evaporation.

Comparing now the classical and quantal simulations, one can see several differences. First of all, it is clear that the effect of entropy (or temperature) is weaker in semi-classical simulations when the excitation is not too high. In particular the stationary points do not exhibit the same entropy (or temperature) dependence in the quantum case or the classical case (see. Fig. 8).

In a similar manner, the initial densities for which the central density will eventually reach 0 (i.e. either the nucleus vaporizes or leads to an hollow system) have different temperature dependence(see Fig.9). At low temperatures, semi-classical approaches are associated with a fast emission of particles. This faster evaporation as compared with the quantum dynamics induces a faster damping of the collective motion. Moreover, it is known that the fluctuations induced by test particle methods as well as the possible turbulent dynamics of classical fluids generate an additional damping of collective motions in semiclassical approaches 25]. All these different dampings reduce the amplitude and the frequency of vibrations. In such a way, a more compressed nucleus can survive more easily in semi-classical simulations because it can dissipate energy more efficiently than its quantum analogue and has a longer time to cool down. At high temperature, all particles are immediately evaporated in the semi-classical approaches leading to the vaporization of the nucleus.

If we now look at the slopes of $\rho_{\text {min }}$ versus $\rho_{i n i}$ in the right part of figure 7 , we can see that the slopes appear smaller in semi-classical case than in both quantum cases. This behavior indicates a larger damping of the collective motion together with a softer response to an external compression of the

nuclei in the semi-classical treatment, compared with the quantum ones. This 


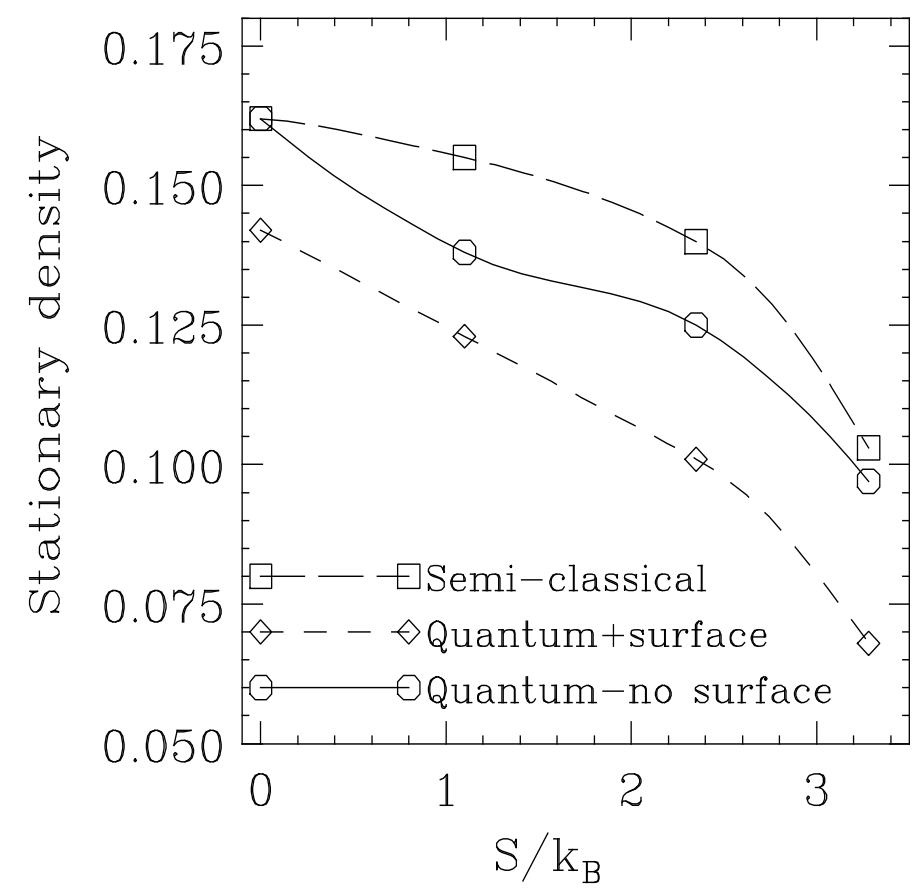

Figure 8: Stationary points obtained from the $\left(\rho_{\text {min }}, \rho_{\text {init }}\right)$ diagram. The stationary density value is plotted as a function of the entropy. Solid-line: Quantum (without surface). Dashed-line: Quantum+Surface. Long dashed line: Semi-classical. 


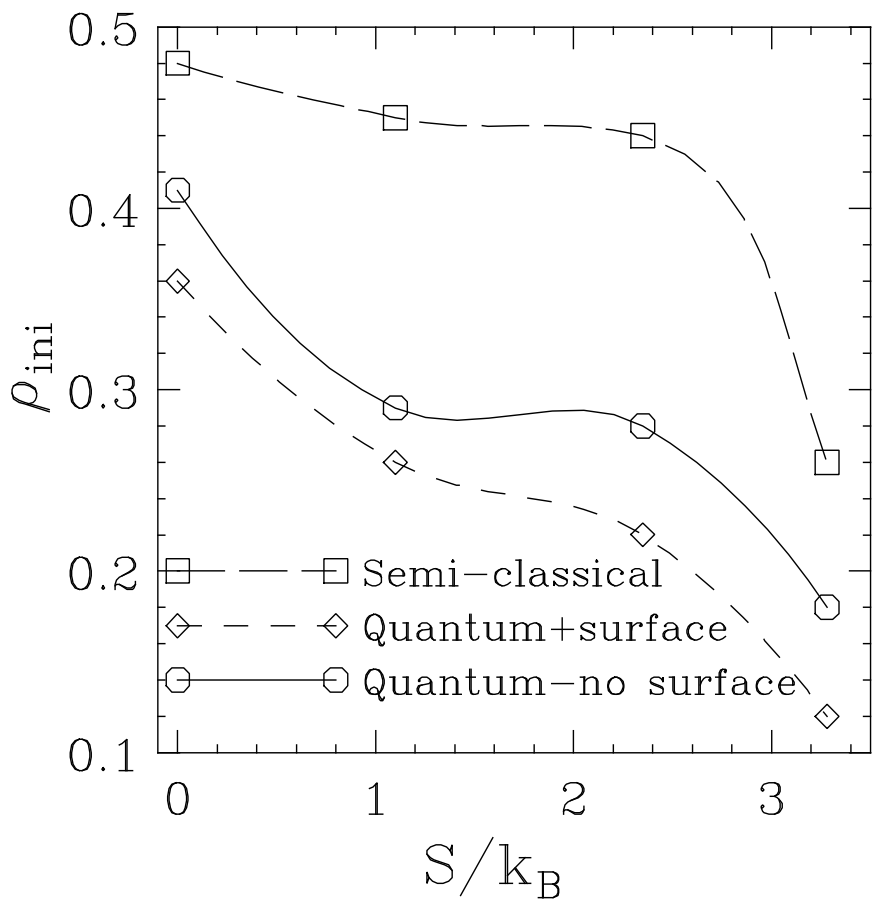

Figure 9: Initial density for which the central density reach 0 during the evolution (from the $\left(\rho_{\text {min }}, \rho_{\text {init }}\right)$ diagram). The initial density value is plotted as a function of the entropy. Solid-line: Quantum (without surface). Dashedline: Quantum+Surface. Long dashed line: Semi-classical. 
apparent softness could appear surprising a priori since we are considering models with same EOS for the infinite nuclear matter. However, we have seen in the previous section that the EOS for finite systems are different in the various simulations (see Fig. 3).

In conclusion, a higher compression is needed in semi-classical treatments as compared with quantum approaches in order to reach the same minimum density. This corresponds to a smaller amplitude and a slower collective motion. Part of this difference could be explained by the smoothing procedure necessary in semi-classical method to generate the mean-field. However, adding a surface term in quantum mechanics reduce only a little this difference, indicating that physical aspect such as, for instance, dampings, evaporation, time-scale... are different. In the following, we will quantify differences in time-scale and in the evaporation process.

\subsubsection{Monopole vibration period}

In the previous section, we have mentioned that semi-classical methods lead to much softer response of the nucleus. As a direct consequence, we could expect a slower expansion dynamics in the semi-classical treatment. In Fig. 10 , we have plotted the time (noted $t_{\text {min }}$ ) necessary to reach the lowest central density $\rho_{\min }$ for isoentropic initial conditions. At high initial compression, this time goes to infinity since the system is vaporized. For initial dilatation below the stationary point, this time is simply equal to zero(or nearly zero) since the system immediately contracts. Inbetween, we have finite, nonzero $t_{\text {min }}$ which could be interpreted to be one-half of the period of monopole vibration. In the two quantum cases, at temperature or entropy equal to zero, the monopole vibration period is close to $80 \mathrm{fm} / \mathrm{c}$ and appears to be almost independent of the initial perturbation (which is similar to the harmonic picture). In the classical case, the period of monopole vibration is of the order of $120 \mathrm{fm} / \mathrm{c}$. This indicates again a softer response of the classical system accompanied with a longer time of expansion. If we now look to higher temperature (or entropies), we see that in quantum cases, the monopole motion period increases with temperature (or entropy) and that for a given temperature or entropy, this time increases with the initial compression. The situation is not as clear in the semi-classical case mainly due to the presence of large fluctuations in the central density which makes the extraction of the corresponding times difficult.

Therefore, as far as the collective motion is concerned, we can conclude 


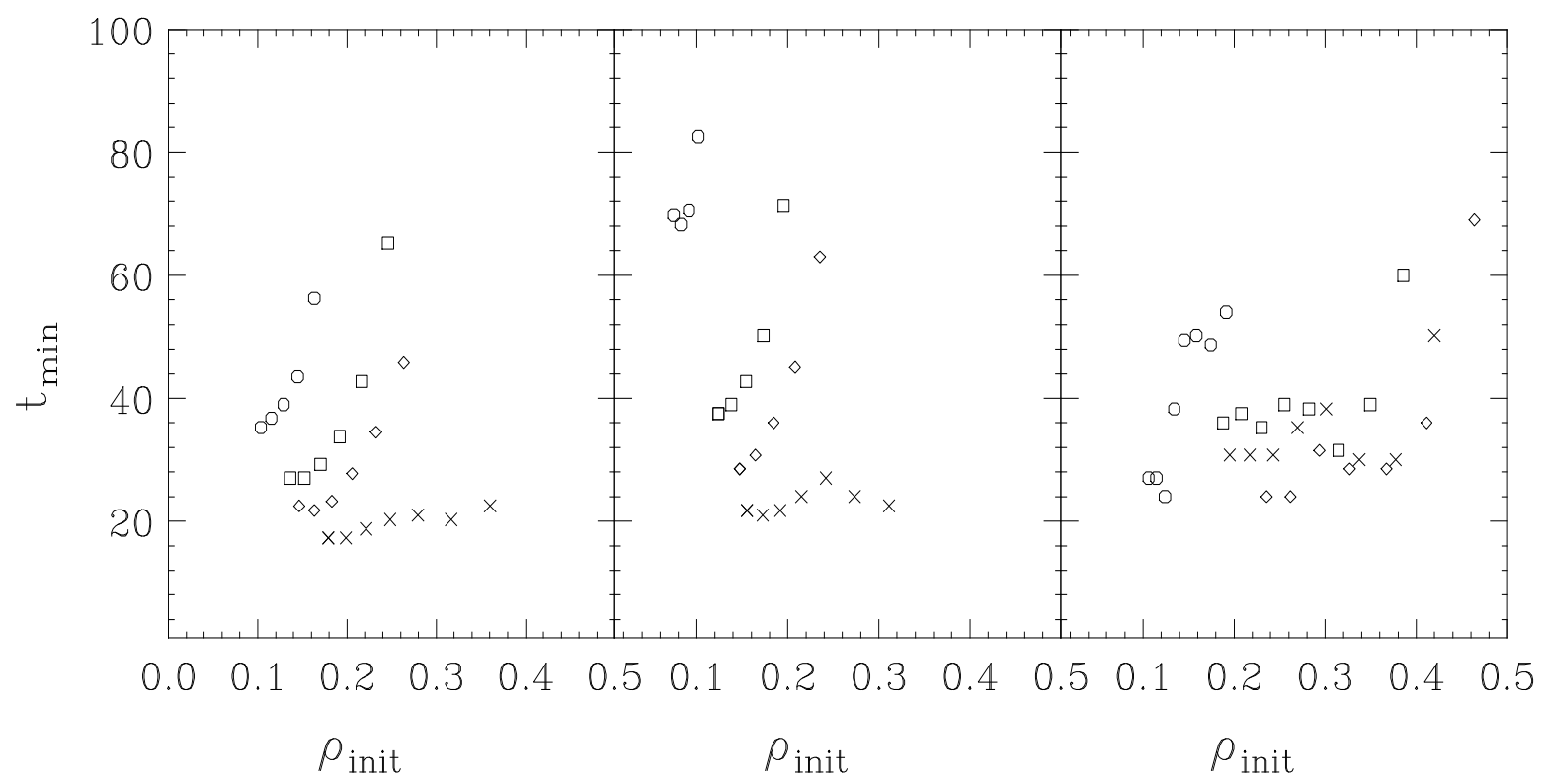

Figure 10: Time $t_{\min }$ to reach the minimal density as a function of the initial density $\rho_{\text {init }}$ at various initial entropies $S / k_{B}=0(\times), 1.1(\diamond), 2.35(\square)$ and 3.28 (०). Left: Quantum (without surface). Middle: Quantum+Surface. Right: Semi-classical simulation. Note that, near the stationnary point, the time $t_{\min }$ could not be extracted in semi-classical simulation due to the strong fluctuations induced by test-particle method (see figure 6). These points are thus not represented in the figure. 
that, compared to the semi-classical predictions, the quantum simulations present a faster expansion towards lower densities. These differences appear to be due to the differences in the collective potential but also to the differences in the damping and cooling processes which appear faster in the semi-classical case. Then, in order to conclude this comparison let us discuss in more details the evaporation process.

\subsubsection{Evaporation dynamics and time scales}

In order to study the evaporation process we have computed the particle flows outside of a sphere of radius $15 \mathrm{fm}$. These flow are shown on figure (11).

According to this figure it is clear that the semi-classical approximation predicts a quicker evaporation of more particles than the quantum one. We see that these differences in the evaporation process are reduced by the introduction in the quantum simulation of a surface interaction (see right part of Fig.11]. This can be clearly quantified by the asymptotic number of evaporated particles (after $300 \mathrm{fm} / \mathrm{c}$ ) which is presented in Fig. 12 as a function of the temperature/entropies for different simulations. We can see that the temperature acts completely differently in quantum and semi-classical cases. In particular for high temperature, the semi-classical model is poor approximation of the quantum mean-field theory. The heated nucleus appears less bound in semi-classical than in quantum cases. However, we note that the introduction of a surface term reduces quantitatively the gap between the two treatments. Nevertheless, the time scales of the evaporation are different.

This important difference can be traced back to the fact that in a quantum approach the nucleons can be partly reflected by the mean-field potential wells while in a classical case as soon as a particle as enough energy it will be immediately emitted. This large difference between semi-classical and quantum approaches is one of the major drawback of semi-classical approximation. Indeed, it affects not only the evaporation itself but in fact the whole dynamics changing time-scales, cooling processes, influence of the thermal pressure and also the one-body dissipation. 


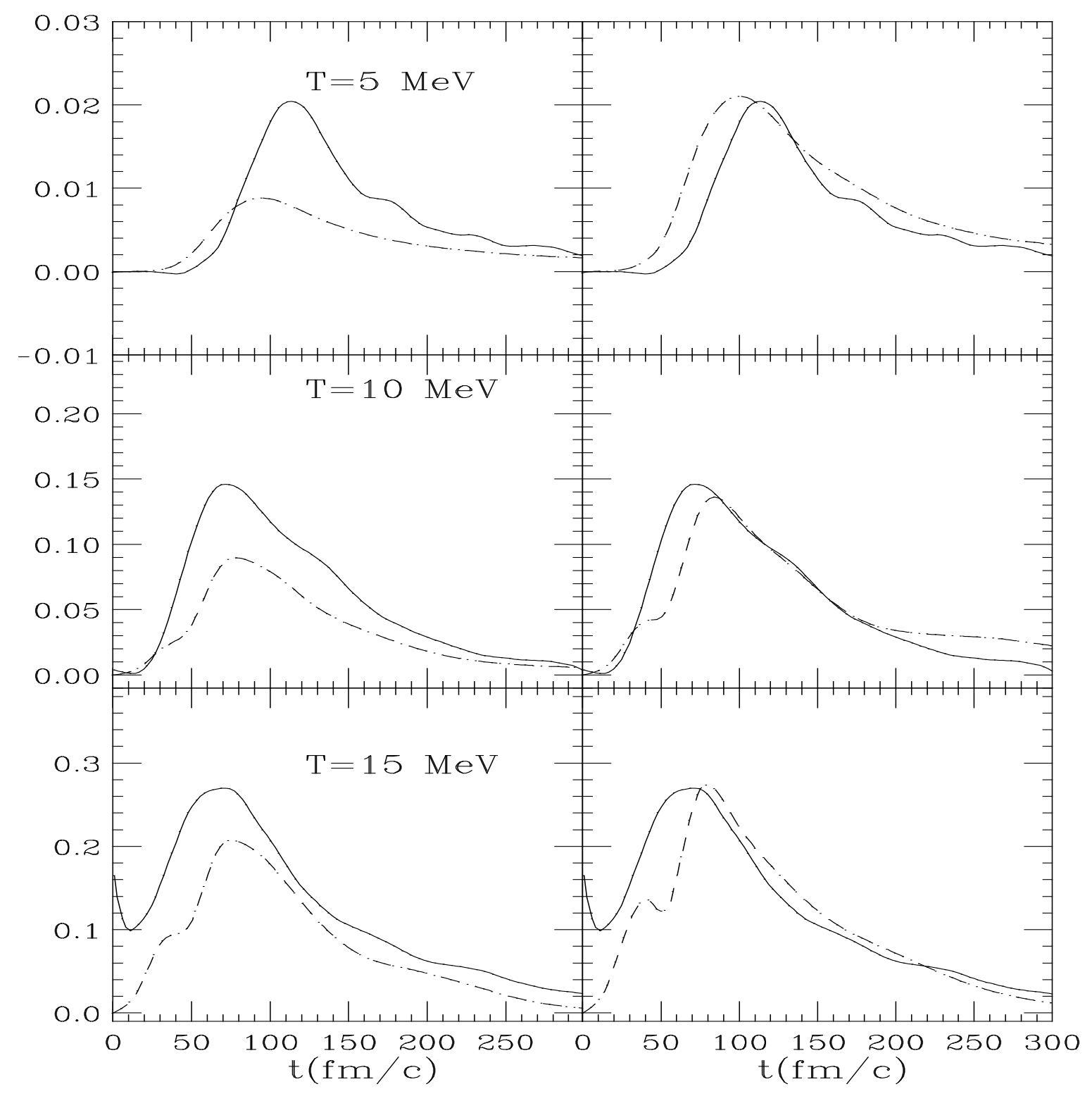

Figure 11: Particle Flow out of a sphere of radius $15 \mathrm{fm}$ for a typical Ca nucleus at various temperatures $T=5,10$ and $15 \mathrm{MeV}$ (from top to bottom). Left: Quantum without surface (dashed line). Right: Quantum+Surface (dashed line). On each figures is superimposed the Semi-classical case (solid line). 


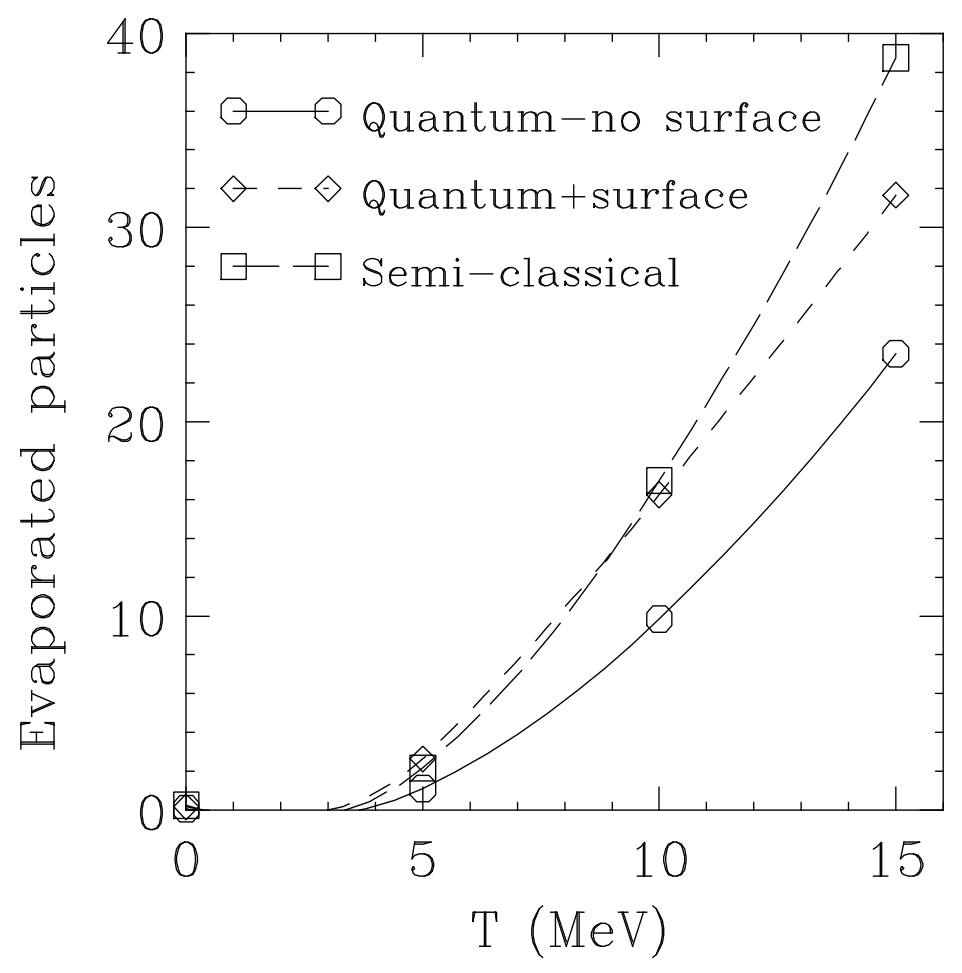

Figure 12: Asymptotic number of evaporated particles (after $300 \mathrm{fm} / \mathrm{c}$ ) as a function of the temperature for the different considered models. Solid line: Quantum without surface. Dashed line: Quantum+Surface. Long-dashed line: Semi-classical. 


\section{Discussion of the expansion and spinodal instabilities}

Semi-classical simulations (including fluctuations) of the spinodal decomposition are able to reproduce globally the experimental fragment partitions 19, 18. However, these simulations seem to underestimate the kinetic energies of the fragments. This is a general observation made on BUU simulations that the expansion velocity at the entrance of the spinodal region is barely enough compared with the experimental data leaving small room for additional slowing down during the fragment formation. It is thus important to discuss the differences observed between semi-classical and quantum dynamics when the nuclei reach low density regions.

At zero temperature, we have seen that the infinite system becomes unstable when the density is less than $0.1 \mathrm{fm}^{-3}$.

From figure 13, it is clear that higher densities (up to a factor 2) are needed in semi-classical calculations to reach a density below $0.1 \mathrm{fm}^{-3}$. If we look now at figure 14, we see that the time needed to reach the low densities is also longer in semi-classical simulation. This should be related to the observed stronger damping of collective motion in the Vlasov case. The induced delay of a factor 1.5 to 2 is associated with a reduction of the collective expansion velocity by the same amount. This may explain why semi-classical simulations taking into account the fragment formation dynamics seem to underestimate the experimental kinetic energies. From this study, it is now clear that quantal simulations are important when one wants to discuss quantitatively collective expansions and fragment formation that could occur during Heavy Ion reactions.

\section{Concluding remarks}

This work presents a comparison between quantum and semi-classical meanfield dynamics of hot and compressed nuclei.

We have first pointed out some properties of the semi-classical approaches using test particle sampling of the phase space. One particularity of this method is the smoothing or the coarse-graining of the phase space in order to define the density. This smoothing adds a finite range term to the initial potential (expression (32)). This numerical surface term affects static and 


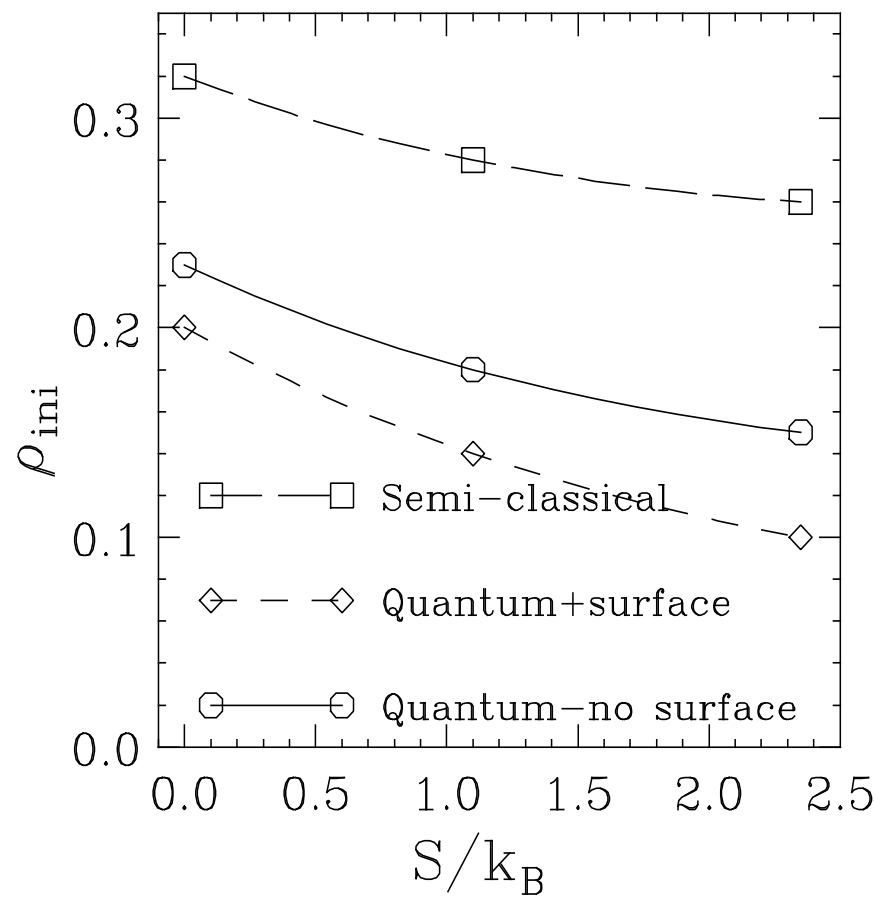

Figure 13: Initial density that gives a minimal density equal to $0.1 \mathrm{fm}^{-3}$ as a function of the entropy. Solid-line: Quantum (without surface). Dashed-line: Quantum+Surface. Long dashed line: Semi-classical. 


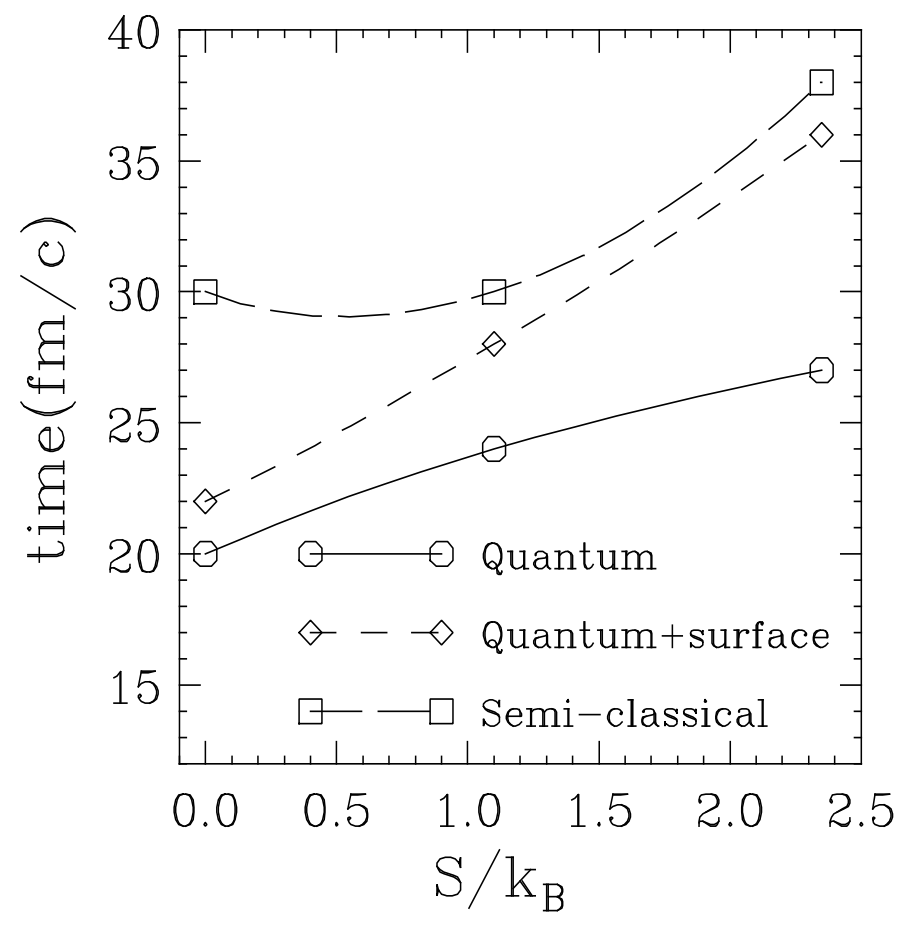

Figure 14: Time necessary to reach a minimal density equal to $0.1 \mathrm{fm}^{-3}$ as a function of the entropy (for the particular initial density that has the minimal density at $0.1 \mathrm{fm}^{-3}$. Solid-line: Quantum (without surface). Dashed-line: Quantum+Surface. Long dashed line: Semi-classical. 
dynamical properties of nuclei. We have disentangled effects due to this extra surface term from intrinsic differences due to the absence of quantum features in semi-classical simulation by comparing them with quantum calculations in which a short range potential was added in order to mimic the numerical one present in semi-classical approaches.

From these three different types of simulations (semi-classical, quantum with and without finite range interaction) we have drawn the conclusions listed bellow.

- While the three approaches have identical static properties of infinite nuclear matter, the energies of finite size nuclei at different dilutions and excitations are different. In particular, the compressibility modulus (or equivalently the curvature of the EOS) of the finite systems depends upon the considered approach. This directly affects the collective dynamics in nuclei. In particular, we have shown that the frequencies of the breathing mode, the dependence in temperature of the amplitude of the collective modes and the position of stationary points are affected by the presence of a numerical gradient of density in the potential. However, the presence of this numerical surface term do not explain all the observed differences and part of the discrepancy is also due to the semi-classical approximation.

- From the dynamical evolutions, we can also see that the damping of the collective mode is greater in semi-classical method than in quantum approaches despite the absence of Landau spreading of the breathing mode 21] in semi-classical approaches. This strong damping observed in semi-classical simulations reflects the differences in the evaporation process as well as additional dissipation in the classical motion of particles. In the semi-classical case, highly excited particles are emitted early in the evolution inducing a large energy loss during the initial stage of the dynamics. This implies a fast attenuation of the collective mode. Conversely, in the quantum case, the reflections of the single-particle wave functions on the potential well reduce the particle evaporation rate and increase the pressure on the nucleus boundary. This difference on time scale is smaller but remains important when a surface interaction is introduced in the quantum-treatment. The evaporation is faster and the total number of emitted particles is larger in semi-classical treatments compared with quantum approaches. 
- The differences in the evaporation process not only affect the damping of the monopole vibration but they also change the size of the remaining residue after desexcitation. At low temperature but extreme conditions of compression, the fast evaporation observed in semi-classical approaches gives a strong loss of energy that enable the system to survive. On the contrary, at high energy, in the semi-classical case, the potential is not able to retain the particles inside the nucleus: the system is directly vaporized. In the quantum case, at low temperature, the collective motion is less damped so that the expansion leads more easily to a complete dilution of the system. Conversely, at high temperature, the evaporation is reduced by the barrier reflections so that a non compressed nucleus may survive the evaporation stage. In summary, in quantum approaches the mean-field is more robust.

- Finally, we have discussed that in a semi-classical treatment the expansion is slower and that stronger compression are needed in order to reach low density regions. This induces an underestimation of the expansion velocity by a factor 1.5 to 2 . This may fill the gap between theoretical prediction of fragment kinetic energies and experimental data since up to now stochastic mean-field description of multifragmentation have been always performed using a semi-classical approximation.

\section{Acknowledgements}

We thank D. Durand for helpful discussion about the physical content of the paper.

\section{References}

\section{References}

[1] G.F. Bertsch, H. Kruse and S. Das Gupta, Phys Rev C29 (1984) 673.

[2] G.F. Bertsch and S. Das Gupta, Phys. Reports 160 (1988) 198.

[3] A. Bonasera et al, Phys. Reports 243 (1994) 1.

[4] C. Gale and S. Das Gupta, Phys. Lett. 162B (1985) 35.

S. Das Gupta, C. Gale and J. Gallego, Phys. Rev. C33 (1986) 1634. 
[5] C. Gregoire et al, Nucl. Phys. A465 (1987) 317.

L. Vinet et al, Nucl. Phys. A468 (1987) 321.

[6] M. Tohayama and E. Suraud, Nucl. Phys. A549 (1992) 461.

[7] W. Bauer et al, Phys. Rev. Lett. 58 (1987) 863.

[8] Y. Abe et al, Phys. Reports 275 (1996) 49.

[9] S. Ayik and C. Gregoire, Phys. Lett. B212 (1988) 269; Nucl Phys. A513 (1990) 187.

J. Randrup and B. Remaud, Nucl. Phys. A514 (1990) 339.

[10] A. Bohr and B. Mottelson, Nuclear physics, (1969), Benjamin N.Y, Nuclear structure, (1975), Benjamin N.Y.

[11] H.H.K Tang et al, Phys. Lett. 101B (1981) 10.

C. Gregoire et al, "Phase-space approach to nuclear dynamics" (Word Scientific 1986) 120.

C. Gregoire, Proceedings of Les Houches Winter School, Amsterdam: North-Holland, 1989.

[12] J. Pochodzalla et al, Phys. Rev. Lett. 75 (1995) 1040. For a review see J. Pochodzalla, Prog. Part. Nucl. Phys. 39 (1997) 443. (and references therein)

[13] D. Vautherin, J. Treiner and M. Veneroni, Phys. Lett. B191 (1987) 6.

[14] D. Vautherin and D. M. Brink, Phys. Rev. C5 (1972) 626.

[15] J.-P. Blaizot, Phys. Rep. 64 (1980) 171.

M. Brack et al, Phys. Rep. 123 (1985) 275.

[16] R. J. Lenk and R. Pandharipande, Phys. Rev. C39 (1989) 2242.

[17] S. E. Koonin, Computational Physics, (1986), Addison-Wesley Publishing Company, Inc., 196 and references therein.

[18] A. Guarnera Thesis, (1996) G.A.N.I.L Caen.

A. Guarnera, M. Colonna and P. Chomaz, Phys. Lett B373, (1996) 267.

[19] E. Suraud et al, Nucl. Phys. A519 (1990) 171c; Nucl. Phys. A452 (1992) 141. 
[20] G. Bertsch and P.J. Siemens, Phys. Lett. 126B (1983) 9.

J. Cugnon, Phys. Lett. 135B (1986) 374 (and ref. therein.)

W. A. Friedman, Phys. Rev. Lett. 60 (1988) 2125; Phys. Rev. C 42 (1990) 667.

[21] P. Chomaz et al, Phys. Lett. B189 (1987) 375.

N. Van Giai et al, Nucl. Phys. A482 (1988) 437c.

[22] E. Lifshitz and L. Pitaevskii, Physical Kinetics, NY: Pergamon, 1981.

[23] M. Colonna and P. Chomaz, Phys. Rev. C49 (1994) 1908.

[24] P. Bonche, S. Koonin and J.W. Negele, Phys. Rev. C13 (1976) 1226. K.T.R. Davies and S.E. Koonin, Phys. Rev. C23 (1981) 2042. S.E. Koonin and J.W. Negele, Phys. Rev. C15 (1977) 1359.

[25] P.G.Reinhard and E. Suraud, Ann. Phys. 239 (1995) 193; Ann. Phys. 239 (1995) 216. 